\title{
Commutation Rules and Spurious Eigenstates in Soluble Field Theories
}

\author{
R. E. Norton and W. K. R. Watson \\ Norman Bridge Laboratory of Physics, California Institute of Technology, Pasadena, California \\ (Received April 13, 1959; revised manuscript received August 31, 1959)
}

\begin{abstract}
A study is made of the exactly soluble field theories which are characterized by Hamiltonians quadratic in the field variables. As an example of such a theory, a model describing the electric dipole interaction of photons and a nonrelativistic, harmonically bound electron is studied explicitly. For the cases when the cutoff is sufficiently large to admit the "runway solutions," it is necessary in order to obtain consistency and a sensible physical interpretation that the theory be reformulated by a unique modification of the equaltime commutation rules of the field operators. The problems which arise here in connection with the "runaway solutions" are closely related to the troubles of ghost states and negative transition "probabilities" which have been demonstrated or suggested to exist in other theories. It is hoped, therefore, that the procedure of reformulation required here may be a guide for the eventual resolution of the ghost-state problem should it be demonstrated that such problems actually exist in the physical, relativistic field theories.
\end{abstract}

\section{INTRODUCTION}

$\mathrm{R}^{\mathrm{E}}$ ECENTLY there has arisen a considerable amount of evidence to indicate that field theories in the limit of no cutoff possess properties which are contrary to those usually assumed in quantum mechanics. In the Lee model, ${ }^{1}$ for example, it has been demonstrated ${ }^{2}$ that the bare coupling constant must be pure imaginary with the result that the Hamiltonian for the theory is not Hermitian. This feature then necessitates a quantization procedure employing a Hilbert space with an indefinite metric and, more important, causes considerable difficulty in the physical interpretation of the theory. Spurious eigenstates of the Hamiltonian appear and the transition processes involving these states occur with a nonpositive "probability." Except for one case ${ }^{3}$ which relies for its success upon the choice of specific values of the parameters of the model, the Lee model has apparently not been interpreted in a manner which is free of ambiguities.

The problems which can be seen so clearly in the Lee model may well be symptomatic of a disease underlying all of field theory. Ambiguities similar to those present in the Lee model have already been shown to exist in a truncated version of relativistic quantum electrodynamics, ${ }^{4}$ and although it has not been proved, they may well be present in the full theory. In any case it is clearly desirable to acquire a better understanding of the problems present in as many theories as possible so that they may be compared and then perhaps resolved in some general manner.

It is with this motivation that we continue here to examine the structure of some exactly soluble field theories. Explicitly we study the model of a nonrelativistic, harmonically bound electron coupled to the radiation field in the electric dipole approximation. We wish to emphasize, however, that essentially the same

\footnotetext{
1 T. D. Lee, Phys. Rev. 95, 1329 (1954).

${ }^{2}$ G. Källén and W. Pauli, Kgl. Danske Videnskab. Selskab, Mat.-fys. Medd. 30, No. 7 (1955).

${ }^{3}$ W. Heisenberg, Nuclear Phys. 4, 532 (1957).

${ }^{4}$ Landau, Abrikosov, and Halatnikov, Suppl. Nuovo cimento'3, 80 (1956).
}

conclusions can be drawn from a study of the pair theory, ${ }^{5}$ or probably, for that matter, from the study of any theory whose Hamiltonian is quadratic in the field variables. We have chosen to study the harmonic oscillator model explicitly, rather than for example the pair theory, simply because it has been the subject of greater interest in the past and because it is at least approximately descriptive of an actual physical interaction.

It has been known for some time that the classical equations of motion for the harmonic oscillator system have unphysical "runaway solutions," 6 and it has been shown by Dirac ${ }^{7}$ that these solutions can only be ignored, and the model thereby made reasonable, by allowing the theory to become acausal over extremely small space-time dimensions. Similarly, in the quantum mechanical formulation of the theory, the equations of motion for the field operators admit these exponentially time-dependent solutions. In analogy to Dirac's prescription in the classical theory, it has been suggested ${ }^{8}$ that the canonical coordinates associated with the imaginary frequency be deleted from the Hamiltonian, and the remaining, truncated version of the theory be quantized in the usual manner.

In this paper we study in somewhat greater detail those aspects of the quantized harmonic oscillator model which arise in conjunction with the "runaway solutions." We conclude, as was done before, ${ }^{8}$ that we must project out from the Hilbert space those states which generate the exponential time dependence. By so doing, however, it is clear that the theory is altered from the one which is defined originally in the usual formulation of the model. In particular, we find that the modification of the theory implied by this truncation process manifests itself as an alteration in the equal-time commutation properties of the field operators. They are no longer the usual delta function ex-

\footnotetext{
${ }^{5}$ G. Wentzel, Helv. Phys. Acta 15, 111 (1942).

${ }^{6}$ See, for example, W. Wessel, Z. Physik 92, 407 (1934).

7 P. A. M. Dirac, Proc. Roy. Soc. (London) 167, 148 (1938).

${ }^{8}$ N. G. Van Kampen, Kgl. Danske Videnskab. Selskab, Mat.fys. Medd. 26, No. 15 (1951).
} 
pressions. On the other hand, the field equations satisfied by these operators are unchanged. The variations in the commutation rules are of such a form that they express the acausality of the theory which arises when the unphysical states are eliminated. This acausality occurs in a manner similar to the lack of causality previously noticed by Dirac. ${ }^{7}$

In Sec. II the theory defined by the equations of motion and the usual commutation rules is solved exactly. We formulate the theory with a cutoff by spreading out the electron over a region determined by a form factor $\rho(x)$. The no-cutoff limit is obtained by allowing $\rho(x)$ to approach a delta function. It is demonstrated anew ${ }^{8}$ that as the cutoff increases, the bare electron mass $m_{0}=m-\delta m$ decreases, and eventually goes to negative infinity in the limit of a point electron. For values of the cutoff larger than the value which gives rise to a zero bare mass, a continuum of new eigenstates of the Hamiltonian appears, and those parts of the field operators which connect to these states are characterized by a real, exponential time dependence. This situation is the quantum analog of the existence of the "runaway solutions" in the classical theory.

In Sec. III the spectrum of the Hamiltonian is discussed and it is argued that the continuum of eigenstates which generate the exponential time dependence should simply be omitted from the Hilbert space. In Sec. IV the theory is then reformulated with equaltime commutation rules modified in such a way that the unphysical, spurious states do not arise. Finally, in Sec. $V$ the work of the preceding parts of the paper is summarized, and the possible generalization of these results is discussed.

It should be emphasized that the acausality which arises in the theory studied here is of exactly the same origin as the acausality which has been known to exist in the corresponding classical problem. Furthermore, the procedure of simply eliminating the spurious eigenstates is essentially a quantum mechanical method of accomplishing Dirac's prescription of ignoring the "runaway solutions" in the classical problem. This procedure has previously been suggested ${ }^{8}$ as the method for dealing with these solutions in the quantized version of the oscillator theory. It is interesting, therefore, that as mentioned previously, exactly the same kind of problems occur in the pair theory and probably also in all theories whose Hamiltonians are quadratic in the field variables. In the pair theory, for example, the spurious states occur when the bare coupling constant is forced negative by increasing the cutoff. The properties of these states and the method of ignoring them are exactly the same as in the Dirac harmonic oscillator model.

Finally, let us remark that at the present time we do not know to what extent the problems of the theory studied here are similar to those which appear to exist in the relativistic field theories. It is planned to investigate the existence of such an analogy and to determine whether the methods for interpreting the oscillator model can eventually be extended for application to more physical theories. The fact that this situation would imply a lack of microscopic causality does not immediately rule it from physical interest. In this regard, we mention the work of Feynman and Wheeler ${ }^{9}$ where it was concluded that the lack of causality present in the classical version of the oscillator problem is not cumulative in the sense that it is capable of adding up to macroscopic intervals in bulk matter. As long as such an addition does not occur, this lack of causality cannot be ruled out on the grounds that it contradicts experience.

\section{EXACT SOLUTION OF THE MODEL}

The Hamiltonian for the theory is ${ }^{10}$

$$
\begin{aligned}
H=\frac{\left[\mathbf{p}(t)-e \int \rho(x) \mathbf{A}(x, t) d^{3} x\right]^{2}}{2(m-\delta m)}+\frac{1}{2} K \mathbf{r}^{2}(t) \\
\quad+\frac{1}{2} \int d^{3} x\{\mathbf{\Pi} \cdot \mathbf{\Pi}(x, t)+\nabla \mathbf{A} \cdot \nabla \mathbf{A}(x, t)\},
\end{aligned}
$$

which describes the interaction of the photon field $\mathbf{A}$ with an electron bound harmonically to a center of force with a strength determined by the spring constant $K$. (We take $\hbar=c=1$ throughout.) A cutoff is introduced by spreading out the charge distribution of the electron over a region characterized by the spherically symmetric form factor $\rho(x)$. Subsequently this cutoff can be removed by allowing $\rho(x)$ to approach a delta function $\delta(x)$. The electric dipole approximation ${ }^{11}$ is apparent since the coupling contains no reference to the electron's position $\mathbf{r}(t)$. The Hamiltonian in Eq. (1), together with the usual equal-time commutation relations

$$
\begin{gathered}
{\left[\mathbf{r}_{j}(t), \mathbf{p}_{\kappa}(t)\right]=i \delta_{j k},} \\
{\left[\mathbf{A}_{j}(x, t), \mathbf{\Pi}_{\kappa}\left(x^{\prime} t\right)\right]=\left[\mathbf{A}_{j}(x, t), \partial_{t} \mathbf{A}_{\kappa}\left(x^{\prime}, t\right)\right]} \\
=i \delta_{j k} \delta^{3}\left(\mathbf{x}-\mathbf{x}^{\prime}\right),
\end{gathered}
$$

infer the equations of motion

$$
\begin{aligned}
\left(m \partial_{t}{ }^{2}+K\right) \mathbf{r}(t) & =-e \int \rho(x) \partial_{t} \mathbf{A}(x, t) d^{3} x+\delta m \partial_{t}{ }^{2} \mathbf{r}(t), \\
\square^{2} \mathbf{A}(x, t) & =\left(\nabla^{2}-\partial_{t}{ }^{2}\right) \mathbf{A}(x, t)=-e \rho(x) \partial_{t} \mathbf{r}(t) .
\end{aligned}
$$

At this stage it is interesting to note that the theory can be defined in terms of either the Hamiltonian or the field equations in conjunction with the commutation

\footnotetext{
${ }^{9}$ J. A. Wheeler and R. P. Feynman, Revs. Modern Phys. 17, 157 (1945).

10 Throughout this paper we completely ignore the gauge condition $\boldsymbol{\nabla} \cdot \mathbf{A}=0$. Since our aim is to examine the structure of a field theory rather than to describe the interaction of light, we feel that this neglect is of no essential consequence.

${ }^{11}$ See, for example, L. I. Schiff, Quantum Mechanics (McGrawHill Book Company, Inc., New York, 1949).
} 
rules. We shall adopt the latter point of view as being the more fundamental, since our aim is to show that the theory defined by Eqs. (2)-(5) is physically inconsistent with our model when the cutoff becomes large.

We shall then attempt to demonstrate that the correct theory is then defined by Eqs. (4) and (5) and by altered versions of the commutation rules (2) and (3).

In order to solve the equations of motion (4) and (5) $\mathrm{we}^{12}$ employ the Fourier transforms

$$
\begin{aligned}
\rho(k) & =\frac{1}{(2 \pi)^{\frac{3}{2}}} \int \rho(x) e^{i \mathbf{k} \cdot \mathbf{x}} d^{3} x \\
\mathbf{A}(k, \omega) & =\frac{1}{(2 \pi)^{2}} \int \mathbf{A}(x, t) e^{i \mathbf{k} \cdot \mathbf{x}-i \omega t} d^{3} x d t \\
\mathbf{r}(\omega) & =\frac{1}{(2 \pi)^{\frac{1}{2}}} \int \mathbf{r}(t) e^{-i \omega t} d t
\end{aligned}
$$

and use them to rewrite the equations in the form

$$
\begin{aligned}
{\left[(m-\delta m) \omega^{2}-K\right] \mathbf{r}(\omega) } & =-i e \omega \int \rho(k) \mathbf{A}(k, \omega) d k, \\
\left(\omega^{2}-k^{2}\right) \mathbf{A}(k, \omega) & =i e \omega \rho(k) \mathbf{r}(\omega) .
\end{aligned}
$$

These equations can be readily solved with the result that for positive frequencies $\omega$

$$
\begin{gathered}
\mathbf{r}(\omega)=\frac{-i e \omega}{D_{()}\left(\omega^{2}\right)} \int \rho(k) \mathbf{A}_{()}(k, \omega) d^{3} k \\
\mathbf{A}(k, \omega)=\int d^{2} k^{\prime}\left(\delta^{3}\left(k-k^{\prime}\right)+\frac{e^{2} \omega^{2}}{\omega^{2}-k^{2}} \frac{\rho(k) \rho\left(k^{\prime}\right) k^{\prime 2}}{D_{()}\left(\omega^{2}\right)}\right) \\
\times \mathbf{A}_{()}\left(k^{\prime}, \omega\right)
\end{gathered}
$$

where we define $\mathbf{A}_{(\text {) }}$ as the solution of

$$
\left(\omega^{2}-k^{2}\right) \mathbf{A}_{()}(k, \omega)=0,
$$

and

$$
D_{( \pm)}\left(\omega^{2}\right)=(m-\delta m) \omega^{2}-K-4 \pi e^{2} \omega^{2} \int \frac{\rho^{2}(k) k^{2} d k}{\omega^{2} \pm i \epsilon-k^{2}} .
$$

The negative-frequency solutions follow immediately from Eqs. (11) and (12) and the condition of Hermiticity $\mathbf{r}(-\omega)[\mathbf{A}(-k,-\omega)]=\mathbf{r}^{*}(\omega)\left[\mathbf{A}^{*}(k, \omega)\right]$. In the usual manner we label the homogeneous solution $\mathbf{A}_{\text {(in) }}, \mathbf{A}_{\text {(out) }}$ when the integrals through singularities caused by vanishing energy denominators are defined by giving the frequency $\omega$ a positive (negative), infinitesimal imaginary part $+i \epsilon$.

By writing $k^{2}=\left(k^{2}-\omega^{2}\right)+\omega^{2}$ in the numerator of the integrand in Eq. (14), we can cancel the first term against the mass correction $\delta m$ so that we are left with a finite integral in the limit of no cutoff, $\rho^{2}(k)=(2 \pi)^{-3}$. The mass correction becomes

$$
\delta m=4 \pi e^{2} \int \rho^{2}(k) d k
$$

and it is clear that for a sufficiently large momentum cutoff the bare mass, $m_{0}=m-\delta m$, becomes negative. For values of the cutoff for which this situation occurs, the $D_{()}\left(\omega^{2}\right)$ function of Eq. (14) has an additional zero for $\omega^{2}=-\omega_{0}^{2}$ (see Appendix A), and there exists another pair of solutions of the field equations, which can be added to the solutions given in Eqs. (11) and (12). Assuming this to be the case $(m-\delta m<0)$, we can write the general solution of field equations (4) and (5) as

$$
\begin{aligned}
\mathbf{r}(t) & =\frac{1}{\left(2 \omega_{0}\right)^{\frac{1}{2}}}\left(\mathbf{O}_{1} e^{-\omega_{0} t}+\mathbf{O}_{2} e^{\omega_{0} t}\right)-i e \int d^{3} k \rho(k)\left(\frac{k}{2}\right)^{\frac{1}{2}}\left[\frac{1}{D_{(+)}\left(k^{2}\right)} \mathbf{a}_{(\mathrm{in})}(k) e^{-i k t}-\frac{1}{D_{(-)}\left(k^{2}\right)} \mathbf{a}_{(\mathrm{in})} *(k) e^{i k t}\right] \\
& =\frac{1}{\left(2 \omega_{0}\right)^{\frac{1}{2}}}\left(\mathbf{O}_{1} e^{-\omega_{0} t}+\mathbf{O}_{2} e^{\omega 0 t}\right)-i e \int d^{3} k \rho(k)\left(\frac{k}{2}\right)^{\frac{1}{2}}\left[\frac{1}{D_{(-)}\left(k^{2}\right)} \mathbf{a}_{(\mathrm{out})}(k) e^{-i k t}-\frac{1}{D_{(+)}\left(k^{2}\right)} \mathbf{a}_{(\mathrm{out})} *(k) e^{i k t}\right],
\end{aligned}
$$

and

$$
\begin{aligned}
\mathbf{A}(k, t) & =-\frac{e \rho(k)}{k^{2}+\omega_{0}^{2}}\left(\frac{\omega_{0}}{2}\right)^{\frac{1}{2}}\left(\mathbf{O}_{1} e^{-\omega_{0} t}-\mathbf{O}_{2} e^{\omega_{0} t}\right)+\int \frac{d^{3} k^{\prime}}{\left(2 k^{\prime}\right)^{\frac{1}{2}}}\left[\left(k\left|\Omega_{(+)}\right| k^{\prime}\right) \mathbf{a}_{(\mathrm{in})}\left(k^{\prime}\right) e^{-i k^{\prime} t}+\left(-k\left|\Omega_{(-)}\right| k^{\prime}\right) \mathbf{a}_{(\mathrm{in})} *\left(k^{\prime}\right) e^{i k^{\prime} t}\right] \\
& =-\frac{e \rho(k)}{k^{2}+\omega_{0}^{2}}\left(\frac{\omega_{0}}{2}\right)^{\frac{1}{2}}\left(\mathbf{O}_{1} e^{-\omega_{0} t}-\mathbf{O}_{2} e^{\omega_{0} t}\right)+\int \frac{d^{3} k^{\prime}}{\left(2 k^{\prime}\right)^{\frac{1}{2}}}\left[\left(k\left|\Omega_{(-)}\right| k^{\prime}\right) \mathbf{a}_{(\mathrm{out})}\left(k^{\prime}\right) e^{-i k^{\prime} t}+\left(-k\left|\Omega_{(+)}\right| k^{\prime}\right) \mathbf{a}_{(\mathrm{out})} *\left(k^{\prime}\right) e^{i k^{\prime} t}\right]
\end{aligned}
$$

where

$$
\left(k\left|\Omega_{( \pm)}\right| k^{\prime}\right)=\delta^{3}\left(k-k^{\prime}\right)+\frac{4 \pi e^{2}}{k^{\prime 2} \pm i \epsilon-k^{2}} \frac{\rho(k) \rho\left(k^{\prime}\right) k^{\prime 2}}{D_{( \pm)}\left(k^{\prime}\right)^{2}}
$$

${ }^{12}$ We employ the methods of A. Klein and B. McCormick, Phys. Rev. 98, 1428 (1955); see also R. Norton and A. Klein, Phys. Rev. 109, 991 (1958). 
and the $\mathbf{a}_{()}(k)$ are defined in terms of the relation

$$
\begin{aligned}
\mathbf{A}_{()}(x, t)= & \frac{1}{(2 \pi)^{\frac{3}{2}}} \int \frac{d^{3} k}{(2 k)^{\frac{1}{2}}} \\
& \left.\times\left[\mathbf{a}_{()}(k) e^{i \mathbf{k} \cdot \mathbf{x}-i \omega t}+\mathbf{a}_{(}\right)^{*}(k) e^{-i \mathbf{k} \cdot \mathbf{x}+i \omega t}\right] .
\end{aligned}
$$

The first terms of Eqs. (16) and (17) contain real exponential time dependence as well as a pair of as yet undefined constant operators $\mathbf{O}_{1}$ and $\mathbf{O}_{2}$. It will be recalled that these parts of the general solution only exist for large cutoff (i.e., $m-\delta m<0$ ), and it will be shown later that those extra solutions must be included if the fields are to satisfy the usual commutation rules, Eqs. (2) and (3).

\section{STRUCTURE OF THE ENERGY SPECTRUM}

If we substitute the relations for $\mathbf{r}(t), \mathbf{A}(k, t)$ from Eqs. (16), (17), and the corresponding expressions for $\mathbf{p}(t), \mathbf{\Pi}(x, t)$ into the Hamiltonian in Eq. (1), we obtain after some lengthy but straightforward manipulations the result

$$
\begin{aligned}
& H=-\frac{\omega_{0}}{2 R}\left(\mathbf{O}_{1} \cdot \mathbf{O}_{2}+\mathbf{O}_{2} \cdot \mathbf{O}_{1}\right) \\
& \left.\left.+\int d^{3} k k\left[\mathbf{a}_{(}\right)^{*}(k) \mathbf{a}_{()}(k)+\mathbf{a}_{()}(k) \mathbf{a}_{(}\right)^{*}(k)\right],
\end{aligned}
$$

where the real number $R$ is defined by

$$
R=\operatorname{limit}_{\omega^{2} \rightarrow-\omega_{0}^{2}} \frac{\omega^{2}+\omega_{0}^{2}}{D_{()}\left(\omega^{2}\right)},
$$

and is hence the residue of $1 / D_{()}\left(\omega^{2}\right)$ at the pole $\omega^{2}=-\omega_{0}^{2}$. In addition to the usual, infinite self-energy, the integral term in Eq. (20) simply sums up the total energy in all the normal modes represented by either the incoming- or outgoing-wave eigenstates. Since these incoming- or outgoing-wave scattering states are all we would ordinarily expect for our model, a question arises about the significance of the first term in Eq. (20).

By insisting that the Hamiltonian in Eq. (20) generate the correct time displacement of the operators $\mathbf{r}(t)$, $\mathbf{A}(k, t)$ given in Eqs. (16) and (17), we obtain the commutation rules

$$
\left[\mathbf{O}_{1}(t), \mathbf{O}_{2}(t)\right]=i R
$$

and, as expected,

$$
\left.\left[\mathbf{a}_{()}(k), \mathbf{a}_{(}\right)^{*}\left(k^{\prime}\right)\right]=\delta^{3}\left(k-k^{\prime}\right) .
$$

Equation (22) can also be obtained directly by the method of Appendix A, reference 12, by insisting that the solutions in (16) and (17) lead to the conventional commutation relations given in Eqs. (2) and (3). Since the first and second terms of Eq. (20) are completely uncoupled, the eigenstates of $H$ are the direct product of the separate eigenstates of each of these two terms in the Hamiltonian. The eigenstates of the right-hand term were discussed above and they have the usual interpretation as the scattering states of the system. On the other hand, the spectrum of the left-hand term in Eq. (20) (see Appendix B) is a continuum over both positive and negative energies and appears to be completely spurious. It is suggested, therefore, that Eqs. (2)-(5) do not correctly describe the oscillator model when the momentum cutoff is larger than the value which gives rise to a bare mass of zero. The theory can, however, be formulated in a consistent manner by simply ignoring the exponential solutions containing $\mathbf{O}_{1}$ and $\mathbf{O}_{2}$. This version of the theory will be presented in the next section.

\section{REVISED FORMULATION OF THE MODEL FOR LARGE CUTOFF}

We have seen that when the momentum cutoff becomes sufficiently large to force $m-\delta m$ negative, there occur an additional pair of solutions of the field equations which are characterized by an unphysical, exponential time dependence. Simultaneously there is introduced an apparently spurious continuum of energy eigenstates. In analogy with the work of Van Kampen, we propose, therefore, that these solutions of the field equations should not be included, and that the correct expressions for the field operators are given by Eqs. (16) and (17) with the first terms containing $\mathbf{O}_{1}$ and $\mathbf{O}_{2}$ absent. The resulting solutions for the field operators and the resulting energy spectrum are then of the same form as would occur if the cutoff were strong enough to forbid the exponential solutions. The interpretation of the theory can then be carried out as usual with the $S$-matrix defined as

$$
\left\langle\mathbf{k}^{\prime} j|S| \mathbf{k} l\right\rangle=\left\langle\mathbf{a}_{j(\text { out })} *\left(k^{\prime}\right) \boldsymbol{\Phi}_{0}, \mathbf{a}_{l(\text { in })} *(k) \boldsymbol{\Phi}_{0}\right\rangle,
$$

where $\left|\boldsymbol{\Phi}_{0}\right\rangle$ is the ground state of the system and represents the physical oscillator with no radiation present. The evaluation of this matrix element is accomplished by equating the two alternative expressions for $\mathbf{A}(k, t)$ given in Eq. (17) (ignoring, of course, the term containing $\mathbf{O}_{1}$ and $\mathbf{O}_{2}$ ) and expressing $\mathbf{a}_{\text {(out) }}$ in terms of

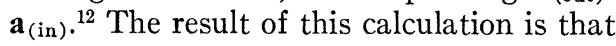

$$
\begin{aligned}
\left\langle\mathbf{k}^{\prime} j|S| \mathbf{k} l\right\rangle=\delta^{3}\left(\mathbf{k}^{\prime}-\mathbf{k}\right) \delta_{j k} e^{2 i \delta} & \\
& =\delta^{3}\left(\mathbf{k}^{\prime}-\mathbf{k}\right) \delta_{j k} D_{(-)}\left(k^{2}\right) / D_{(+)}\left(k^{2}\right),
\end{aligned}
$$

and the unitarity of the $S$-matrix is therefore manifest.

Furthermore, if we follow the usual definition of the scattering amplitude $\left\langle\mathbf{k}^{\prime} j|T| \mathbf{k} l\right\rangle$, namely

$$
\begin{array}{r}
\left\langle\mathbf{k}^{\prime} j|S| \mathbf{k} l\right\rangle=\delta^{3}\left(k^{\prime}-k\right) \delta_{j l} \\
-2 \pi i \delta\left(k-k^{\prime}\right) \delta_{j l}\left\langle k^{\prime} j|T| k l\right\rangle,
\end{array}
$$

we obtain from Eq. (25) that

$$
\left\langle k^{\prime} j|T| k l\right\rangle=\delta_{j l} \frac{2 \pi e^{2} k^{3} \rho^{2}(k)}{D_{(+)}\left(k^{2}\right)},
$$


and it is evident that if $D_{(+)}\left(k^{2}\right)$ has a zero for $k=i \omega_{0}$, the scattering amplitude possesses an additional pole in the upper half complex energy plane. Since the vanishing of $D_{()}\left(k^{2}\right)$ for a negative value of $k^{2}$ is precisely the condition which allows the "runaway solutions," this lack of causality arises in conjunction with the exponential solutions and therefore occurs whenever the cutoff is sufficiently large to imply a bare mass less than zero. Since except for the case of a point electron (no cutoff), the theory is acausal because $\rho(k)$ must have a pole in the upper half $k$ plane, we are forced to conclude that the theory is acausal for all values of the cutoff. If we attempt to remove the acausality due to the nonlocality of the interaction, we ultimately introduce this additional lack of causality which results from the vanishing of $D_{()}\left(-\omega_{0}^{2}\right)$.

We now note, however, that if the exponential terms in the field operators are to be ignored, we must clearly also ignore the contribution of these terms to the commutation rules. By calculations similar to those presented in Appendix A, reference 12, these can be computed to be

$$
\begin{aligned}
{\left[\mathbf{A}_{j}(x, t), \mathbf{\Pi}_{k}\left(x^{\prime}, t\right)\right] } & =i \delta_{j k}\left\{\delta^{3}\left(x-x^{\prime}\right)+R \omega_{0}^{2} e^{2} f(x) f\left(x^{\prime}\right)\right\}, \\
{\left[\mathbf{r}_{j}(t), \mathbf{p}_{k}(t)\right] } & =i \delta_{j k}\left(1+R K / \omega_{0}^{2}\right), \\
{\left[\mathbf{r}_{j}(t), \mathbf{A}_{k}(x, t)\right] } & =-i \delta_{j k} e R f(x), \\
{\left[\mathbf{p}_{j}(t), \mathbf{\Pi}_{k}(x, t)\right] } & =i \delta_{j k} e R K f(x), \\
{\left[\mathbf{r}_{j}(t), \mathbf{\Pi}_{k}(x, t)\right] } & =\left[\mathbf{p}_{j}(t), \mathbf{A}_{k}(x, t)\right]=0
\end{aligned}
$$

where

$$
f(x)=\frac{1}{(2 \pi)^{\frac{3}{2}}} \int \frac{\rho(k) e^{i \mathbf{k} \cdot \mathbf{x}} d^{3} k}{k^{2}+\omega_{0}^{2}},
$$

and approaches a Yukawa function of range $1 / \omega_{0}$ comparable in extent to the classical electron radius in the limit of no cutoff $\left[\rho(k) \rightarrow(2 \pi)^{-3}\right]$.

It is observed, therefore, that we are forced to modify our original formulation of the theory presented in Eqs. (2)-(5), unless, of course, the cutoff is small enough to forbid the exponential solutions, in which case no problems arise and the usual commutation rules can be employed. For the case of interest, namely, large cutoff or the limit of infinite cutoff (no cutoff), we can reformulate the theory by stating the equations of motion given in (4) and (5) together with the commutation rules (28) and (29). It is this formulation of the theory which leads to solutions with no unphysical terms and which hence is capable of interpretation.

Despite the unusual appearance of the commutators in Eqs. (28)-(32), it can readily be verified that, except for the linear momentum, there exist the usual operators (angular momentum, the Hamiltonian, etc.) which generate the symmetry transformations of the theory. All these operators have the usual commutation properties with the field operators. The fact that there exists no operator for the linear momentum of the theory arises because the model is not invariant under spatial displacements. In the case that the methods used here can be generalized to more physical theories which do conserve momentum, such a situation would not be expected to occur.

\section{CONCLUSIONS}

We have seen that the formulation of the quantized Dirac harmonic oscillator model with the usual commutation rules leads to a physical solution only if there exists a small momentum cutoff. For the case of a large momentum cutoff, and in particular for the limit of a local interaction, it is necessary to reformulate the theory by altering the commutation rules. Otherwise we are forced to include additional terms in the solutions for the field operators which have an unphysical, exponential time dependence. At the same point it becomes necessary to change the commutation rules, and mathematically for exactly the same reason, a pole is introduced into the scattering amplitude when the photon energy $k$ is extended to $k=i \omega_{0}$. This source of acausality is present whenever the momentum cutoff is larger than the value which yields zero bare mass, and consequently the theory is not causal even in the limit of a local interaction.

We emphasize that the modified commutation rules, (28)-(32), are essentially uniquely determined by the requirements that the field operators satisfy the equations of motion (4) and (5) and that they do not contain the solutions with the unphysical, exponential time dependence. It is clear from Eqs. (16) and (17) that this uniqueness exists to the extent that the commutation rules for the incoming (or outgoing) fields $\mathbf{a}_{(\mathrm{in})}(\mathbf{k})$ are required to be the usual expressions given in Eq. (23). This choice is certainly the most natural one and, as discussed in Sec. III, leads with the conventional Hamiltonian in Eq. (1) to the familiar interpretation of $\mathbf{a}_{(\text {in) }} *(\mathbf{k}) \mathbf{a}_{(\text {in) }}(\mathbf{k})$ as the number of photons per unit momentum impinging on the oscillator. We therefore adopt the point of view that the freely propagating incoming or outgoing fields must satisfy the usual delta-function commutation rules in analogy with the conventional formulation of a free-field theory, since this technique leads to a simplicity in interpretation which is desirable. Nevertheless, we should point out that this uniqueness does not exist from the purely mathematical standpoint. ${ }^{13}$

The problems which occur in the model discussed here appear to rise because the mass correction is positive and the bare mass is hence forced negative for sufficiently large cutoffs. On the other hand, for the Lee model ${ }^{1-3}$ and for the truncated version of quantum electrodynamics ${ }^{4}$ the troubles which have appeared seem to be associated with the bare coupling constant. Despite this distinction, it is our feeling that the

\footnotetext{
${ }^{13}$ Eugene P. Wigner, Phys. Rev. 77, 711 (1950).
} 
problems demonstrated here for the oscillator model are at least basically of a common origin with those problems which have been demonstrated for these other theories. As we mentioned in Sec. I, the pair theory is characterized by "runaway solutions" in exactly the same fashion as is the model discussed here. In the pair theory, however, the trouble does arise when the coupling constant is forced negative by an increasing momentum cutoff.

Finally, we would like to point out that the existence of the "runaway solutions" is not simply a result of the electric dipole approximation. ${ }^{14}$ In addition, by increasing the spring constant $K$ to enhance the binding of the electron we would expect the electric dipole approximation to be valid over a larger range of momenta. The occurrence of the unphysical solutions, on the other hand, depends only upon $\delta m$ and not upon the spring constant $K$. We might expect, therefore, to be able to make the spring constant sufficiently strong to insure the validity of the electric dipole approximation over the range where $\rho(k)$ contributes and still have this range of sufficient extent to yield $\delta m=4 \pi e^{2} \int \rho^{2}(k) d k$ large enough to yield a negative bare mass. We consequently feel that characteristics of the theory discussed here are at least qualitatively more general than the electric dipole approximation, and in the event that similar problems do actually occur in the relativistic field theories, there exists a reasonable possibility that they will be resolved by a procedure analogous to the method in this paper.

\section{ACKNOWLEDGMENTS}

One of us (R.E.N.) would like to express his gratitude to the California Institute of Technology for the Richard C. Tolman postdoctoral research fellowship which he held during the course of this work. Both of us wish to thank Professors R. Feynman and M. GellMann for many illuminating discussions; and also Mr. Sidney Coleman for a number of enjoyable and helpful conversations.

\section{APPENDIX A}

It will be recalled that

$$
D_{( \pm)}\left(\omega^{2}\right) \mathbf{r}(\omega)=-i e \omega \int \rho(k) \mathbf{A}_{()}(k, \omega) d^{3} k
$$

where

$$
D_{( \pm)}\left(k^{2}\right)=m_{0} k^{2}-K-4 \pi e^{2} k^{2} \int \frac{d k^{\prime} \rho^{2}\left(k^{\prime}\right)}{k^{2} \pm i \epsilon-k^{\prime 2}} .
$$

Extending $D\left(k^{2}\right)$ into the complex plane by letting $z=k^{2}$ and defining $D_{( \pm)}(z)$ as the limit of $D(z)$ as we approach the real axis from above $(+)$ or below $(-)$, we can investigate the zeros of the function in a convenient fashion.

\footnotetext{
${ }^{14} \mathrm{~A}$. Loinger, Nuovo cimento 2, 511 (1955).
}

\section{Zeros of the $D$ Function}

Case $(i): m_{0} \geq 0$, i.e., $\delta m<m$.

Rewriting Eq. (A.2) as a function of $z$, we obtain

$$
D(z)=m_{0} z-K-4 \pi e^{2} z \int \frac{k^{\prime 2} \rho^{2}\left(k^{\prime}\right)\left(\bar{z}-k^{\prime 2}\right) d k^{\prime}}{\left|z-k^{\prime 2}\right|^{2}}
$$

from which it follows that

$$
\operatorname{Im} D(z)=\operatorname{Im}(z)\left(m_{0}+4 \pi e^{2} \int \frac{k^{\prime 4} \rho^{2}\left(k^{\prime}\right) d k^{\prime}}{\left|z-k^{\prime 2}\right|^{2}}\right),
$$

and it is clear that $D(z)$ cannot have any zeros off the real axis if $\operatorname{Im}(z) \neq 0$. Moreover it is apparent from Eq. (A.3) that $D_{( \pm)}(z)$ is real and negative definite for $z$ on the negative real axis, and from Eq. (A.2) that $\operatorname{Im} D_{( \pm)}(z)$ is nonvanishing on the positive real axis. We conclude therefore that $D_{( \pm)}(z)$ has no zeros anywhere in the cut $z$ plane.

$$
\text { Case (ii) : } m_{0}<0 \text {, i.e., } \delta m>m \text {. }
$$

Let us write $m_{0}=-\left|m_{0}\right|$, so that we obtain

$$
\begin{aligned}
\operatorname{Im} D(z)= & \operatorname{Im}(z)\left(-\left|m_{0}\right|+4 \pi e^{2} \int \frac{k^{\prime 4} \rho^{2}\left(k^{\prime}\right) d k^{\prime}}{\left|z-k^{\prime 2}\right|^{2}}\right) \\
\operatorname{Re} D(z)= & -K-4 \pi e^{2}|z|^{2} \int \frac{k^{\prime 2} d k^{\prime} \rho^{2}\left(k^{\prime}\right)}{\left|z-k^{\prime 2}\right|^{2}} \\
& +\operatorname{Rez}\left(-\left|m_{0}\right|+4 \pi e^{2} \int \frac{k^{\prime 4} \rho^{2}\left(k^{\prime}\right) d k^{\prime}}{\left|z-k^{\prime 2}\right|^{2}}\right) .
\end{aligned}
$$

If $\operatorname{Im}(z) \neq 0$, then for $\operatorname{Im} D(z)=0$ we require

$$
-\left|m_{0}\right|+4 \pi e^{2} \int \frac{k^{\prime 4} \rho^{2}\left(k^{\prime}\right) d k^{\prime}}{\left|z-k^{\prime 2}\right|^{2}}=0,
$$

in which case $\operatorname{Re} D(z) \neq 0$. Hence $D(z)$ has no zeros off the real axis.

Let us now look for zeros of $D_{()}(z)$ on the negative real axis. In this case it is convenient to set $z=-x$ so that

$D(-|x|)=\left|m_{0}\right||x|-K-4 \pi e^{2}|x| \int \frac{k^{\prime 2} d k^{\prime} \rho^{2}\left(k^{\prime}\right)}{|x|+k^{\prime 2}}$,

and it is essentially obvious that there exists a zero for some value of $|x|$, say $|x|=\omega_{0}^{2}$, so that $D_{()}\left(-\omega_{0}^{2}\right)=0$. It is also clear from Eq. (A.7) that $d / d|x|(D(-|x|) /|x|)$ $>0$, so that there can only be one value of $z$ for which $D$ vanishes. We may summarize these results by saying that in the event of $m_{0}>0$, there are no zeros of $D_{( \pm)}(z)$ and for $m_{0}<0$ there is one zero along the negative, real $z$ axis. 
APPENDIX B. THE SPECTRUM OF THE UNPHYSICAL PART OF THE HAMILTONIAN

We have seen in Sec. III that the unphysical part of the Hamiltonian in Eq. (20) is given by

$$
H_{0}=-\frac{1}{2} \omega_{0}(\boldsymbol{\alpha} \cdot \boldsymbol{\beta}+\boldsymbol{\beta} \cdot \boldsymbol{\alpha}),
$$

where $[\boldsymbol{\alpha}, \boldsymbol{\beta}]=i$, and where the $\boldsymbol{\alpha}, \boldsymbol{\beta}$ employed here differ from the $\mathbf{O}_{1}, \mathbf{O}_{2}$ in Eq. (20) by the factor $R$ : $\mathbf{0}_{1}=R^{\frac{1}{2}} \boldsymbol{\alpha}, \mathbf{O}_{2}=R^{\frac{1}{2}} 3$.

To obtain the eigenstates of $H_{0}$ given in Eq. (B.1), we employ the familiar representation $\alpha \rightarrow \mathbf{x}, \beta \rightarrow(1 / i) \nabla$, and solve the resulting Schrödinger equation,

$$
\frac{1}{2} i \omega_{0}(\mathbf{x} \cdot \boldsymbol{\nabla}+\boldsymbol{\nabla} \cdot \mathbf{x}) \psi_{E}(x)=E \psi_{E}(x),
$$

with the result that (confining ourselves to one dimension for simplicity)

or

$$
\begin{aligned}
& \psi_{E}(x)=N x^{-\frac{1}{2}\left(1-2 i E / \omega_{0}\right)} \quad \text { for } \quad x>0 \\
& =0 \quad \text { for } x<0 \text {, }
\end{aligned}
$$

$$
\begin{aligned}
& \psi_{E}^{\prime}(x)=0 \quad \text { for } x>0 \\
& =N^{\prime} x^{-\frac{1}{2}\left(1-2 i E / \omega_{0}\right)} \quad \text { for } \quad x<0
\end{aligned}
$$

or a linear combination of both alternatives. $N$ and $N^{\prime}$ are normalization constants adjusted so that

$$
\int_{0}^{\infty} \psi_{E^{\prime}} *(x) \psi_{E}(x) d x=\delta\left(E^{\prime}-E\right),
$$

or

$$
\int_{-\infty}^{0} \psi_{E^{\prime}}{ }^{\prime *}(x) \psi_{E}^{\prime}(x) d x=\delta\left(E^{\prime}-E\right),
$$

depending upon the choice of solution. Since these normalization requirements are only possible for real energies, we conclude that the spectrum of the Hamiltonian is a continuum over all real energies, positive and negative.
An interesting feature of the Hamiltonian (B.1) is that it generates time displacements which are characterized by real time exponentials, despite the fact that $H_{0}$ is Hermitian and hence has real eigenvalues. This rather unusual result can be seen readily if we examine matrix elements of $x(t)$ and $p(t)$ between wave-packet states. It should be emphasized that such matrix elements do not exist between exact eigenstates of the energy and it is for this reason that this apparently paradoxical result can be realized. In particular, if we consider the matrix element

$$
\langle F|x(t)| G\rangle,
$$

where $|F\rangle$ and $|G\rangle$ are wave-packet states defined, for example, by

$$
|F\rangle=\int F(E)\left|\psi_{E}\right\rangle d E,
$$

we obtain

$$
\begin{aligned}
& \langle F|x(t)| G\rangle \\
& =N^{2} \int_{0}^{\infty} d x d E d E^{\prime} x^{i\left[\left(E^{\prime}-E\right) / \omega_{0}\right]} \\
& =N^{2} \int_{0}^{\infty} d x d E d E^{\prime} \\
& \quad \times \exp \left[i\left(E^{\prime}-E\right) t\right] F^{*}\left(E^{\prime}\right) G(E) \\
& \quad \exp i\left\{\left[\left(E^{\prime}-E\right) / \omega_{0}\right] \ln x-\omega_{0} t\right\} F^{*}\left(E^{\prime}\right) G(E) .
\end{aligned}
$$

By changing the $x$ variable of integration to $y=x e^{\omega_{0} t}$, it follows immediately that

$$
\begin{aligned}
\langle F|x(t)| G\rangle= & N^{2} e^{-\omega_{0} t} \int_{0}^{\infty} d y d E d E^{\prime} \\
& \times y^{\left[i\left(E^{\prime}-E\right) / \omega_{0}\right]} F^{*}\left(E^{\prime}\right) G(E) \\
= & e^{-\omega_{0} t}\langle F|x(0)| G\rangle .
\end{aligned}
$$

